\title{
Expectativas sobre o sucesso do tratamento de dependência de drogas psicotrópicos
}

\section{na visão dos especialistas}

\author{
Expectations about the success of treatment for addiction to psychotropic drugs in the view of \\ specialists
}

Expectativas sobre el éxito del tratamiento de la adicción a psicofármacos a juicio de los especialistas

\section{Resumo}

O presente trabalho teve como objetivo compreender como os especialistas, estudiosos da dependência de substâncias psicoativas, consideram o sucesso de um tratamento dessa problemática levando em conta suas vivências, experiências e as suas diferentes abordagens relacionadas ao tema no Brasil. Foram utilizadas as bases da metodologia qualitativa. A construção da amostra foi baseada no princípio da amostra intencional; utilizou-se como ferramenta para essa pesquisa um questionário eletrônico na plataforma Monkey Survey; o recrutamento dos acadêmicos doutores foi feito utilizando a plataforma Lattes do CNPq. O processo de análise dos resultados seguiu as premissas da Análise de Conteúdo: assim o material possibilitou a construção de área temática e a partir do agrupamento dessas informações, foram construídas as categorias para análise. Dessa forma, foram enviados 464 e-mails, e foram considerados 55 participantes, 5 categorias foram encontradas (Mudança no padrão de consumo; Qualidade de Vida; Reinserção Social; Desenvolvimento Emocional; Autonomia). A análise demonstrou que os especialistas têm expectativas muito audaciosas como: abstinência, independência na condução da própria vida, retorno às atividades de trabalho e de estudo, reativar as relações com família e amigos. Essas expectativas são de difícil alcance já que são ignoradas outras variáveis que interferem na concretização das mesmas, como por exemplo: preconceito e rejeição da sociedade a usuários de substâncias, família não amistosa, situação de vulnerabilidade, etc. Esse quadro pode dificultar o processo de tratamento dos indivíduos acometidos pela problemática da dependência de substâncias.

Palavras-chave: Expectativas; Dependência; Tratamento; Metodologia qualitativa.

\begin{abstract}
This study aimed to understand how specialists, scholars of psychoactive substance dependence, consider the success of a treatment of this problem, taking into account their experiences and their different approaches to the subject in Brazil. The bases of qualitative methodology were used. The construction of the sample was based on the principle of intentional sampling; an electronic questionnaire on the Monkey Survey platform was used as a tool for this research; the recruitment of $\mathrm{PhD}$ academics was done using CNPq's Lattes platform. The process of analyzing the results followed the premises of Content Analysis: thus, the material enabled the construction of a thematic area and from the grouping of this information, categories for analysis were constructed. Thus, 464 emails were sent, and 55 participants were considered, 5 categories were found (Change in consumption pattern; Quality of Life; Social Reinsertion; Emotional Development; Autonomy). The analysis showed that specialists have very audacious expectations, such as: abstinence, independence in conducting their own lives, returning to work and study activities, reactivating relationships with family and friends. These expectations are difficult to reach, since other variables that interfere in their realization are ignored, such as: prejudice and society's rejection of substance users, unfriendly family, vulnerable situations, etc. This situation can hinder the treatment process of individuals affected by the problem of substance dependence.
\end{abstract}

Keywords: Expectations; Dependency; Treatment; Qualitative methodology. 


\begin{abstract}
Resumen
Este estudio tuvo como objetivo comprender cómo los especialistas, estudiosos de la dependencia de sustancias psicoactivas, consideran el éxito de un tratamiento de este problema, teniendo en cuenta sus vivencias, vivencias y sus diferentes enfoques del tema en Brasil. Se utilizaron las bases de la metodología cualitativa. La construcción de la muestra se basó en el principio de muestreo intencional; se utilizó un cuestionario electrónico en la plataforma Monkey Survey como herramienta para esta investigación; La contratación de doctores académicos se realizó utilizando la plataforma Lattes del CNPq. El proceso de análisis de los resultados siguió las premisas del Análisis de Contenido: así, el material permitió la construcción de un área temática y a partir de la agrupación de esta información se construyeron categorías para el análisis. Así, se enviaron 464 correos electrónicos y se consideraron 55 participantes, se encontraron 5 categorías (Cambio en el patrón de consumo; Calidad de Vida; Reinserción Social; Desarrollo Emocional; Autonomía). El análisis mostró que los especialistas tienen expectativas muy audaces, tales como: abstinencia, independencia en el desarrollo de su propia vida, reincorporación a las actividades laborales y de estudio, reactivación de las relaciones con familiares y amigos. Estas expectativas son difíciles de alcanzar, ya que se ignoran otras variables que interfieren en su realización, como son: el prejuicio y el rechazo de la sociedad a los consumidores de sustancias, la familia hostil, las situaciones de vulnerabilidad, etc. Esta situación puede dificultar el proceso de tratamiento de las personas afectadas por el problema de la drogodependencia.
\end{abstract}

Palabras clave: Expectativas; Dependencia; Tratamiento; Metodología cualitativa.

\title{
1. Introdução
}

Estima-se que 5,5\% (270 milhões de pessoas) da população mundial já experimentou algum tipo de substância psicotrópica (substâncias psicoativas que atuam no sistema nervoso central e podem causar dependência), e entre essas, 35 milhões necessitam de tratamento em função dos problemas relacionados ao uso dessas substâncias. Por outo lado, dentre essas pessoas, somente $1 \mathrm{em}$ cada 8 consegue receber tratamento para os transtornos relacionados ao uso de drogas, seja por dificuldade de acesso, por estigma ou ainda, por falta de adesão (Tomko et al., 2016; World Drug Report, 2021).

Embora seja pequeno o número de pessoas que usam drogas tornarem-se dependentes (UNODC, 2017), o controle dos problemas originados por conta da dependência tem sido um desafio, levando à utilização de combinações de terapias com medicamentos na busca da abstinência. Apesar de todos os avanços na área farmacológica, proporcionando o aparecimento de novos medicamentos, o resultado não tem mudado ao longo do tempo e as recaídas são uma constante entre os dependentes de drogas (Behere et al., 2009; Lopes-Rosa et al., 2017; Yang et al., 2015).

Tomando o usuário de crack como exemplo, nos seis primeiros meses após o tratamento, as recaídas podem alcançar uma taxa de 80\% (Sadeghieh et al., 2004) ou mesmo 90\% (Yang et al., 2015). Em estudo em Porto Alegre com adolescentes que usavam crack e estavam em abstinência após tratamento, os autores identificaram altas taxas de recaídas nessa população: $69,9 \%$ no primeiro mês e $86,4 \%$ no terceiro (Lopes-Rosa et al., 2017).

Esse modelo biomédico para tratamento de pessoas que usam drogas o qual privilegia a intervenção farmacológica, tem sido preponderante nos serviços públicos e privados de cuidado e atenção ao dependente. Ele parte do princípio que a droga tem o poder de tornar o indivíduo dependente e dessa forma a abstinência é o objetivo que deve ser alcançado. Em suma, o tratamento pela abstinência pauta-se na droga desconsiderando os aspectos individuais, subjetivos, sociais e culturais implicados no fenômeno de dependência (Ribeiro \& Fernandes, 2013).

Esse modelo, embora reconhecido internacionalmente, tem colecionado fracassos quando se dispõe a alcançar a abstinência do uso da droga como principal meta do tratamento. Fato até compreensível quando se considera que após intenso período de uso abusivo da droga, manter-se em total abstinência é supostamente um tremendo esforço para o usuário de substância. Marlatt (1999) considera esse modelo como de alta-demanda. O autor ainda acrescenta que a abstinência pode estar associada com recaída e é a barreira para acessar e/ou dar continuidade ao tratamento.

Porém, há ganhos alcançados através dos tratamentos convencionais que são valiosos para a qualidade de vida da pessoa que usa substância. Por exemplo, um uso controlado da droga facilitaria a reintegração do usuário à sociedade, com a família, trabalho e poderia ser uma estratégia para evitar a recaída. Oliveira e Nappo (2008) identificaram um padrão 
controlado de uso de crack em São Paulo, o qual compreendia um uso da droga em alguns dias na semana o que permitia reconectar o usuário às suas atividades sociais (família, escola, trabalho), protegendo-o da marginalização.

Considerando esses fatos, pode-se inferir que o sucesso no tratamento de dependência pode ter uma definição muito ampla. Há muito tempo que se associa a resolução de problemas graves, decorrentes do abuso de substâncias psicoativas, a diferentes termos, como "reforma" moral. "redenção religiosa", reabilitação criminal ou "recuperação médica'. O termo recovery, recuperação, tem sido empregado tanto por usuários como por profissionais ligados a saúde no Brasil e em diversos países (Inanlou et al., 2020; Laudet, 2007; Rigotto \& Gomes, 2002) para representar o resultado do tratamento de uma doença crônica como a dependência de substâncias (Inanlou et al., 2020). Apesar do uso da palavra recovery, não existe um consenso sobre essa definição a qual pode significar condições diferentes dependendo da visão do profissional. (Center for Substance Abuse Treatment, 2010, 2006; El-Guebaly, 2012; Galanter, 2007; Inanlou et al., 2020; Laudet \& White, 2010; Betty Ford Institute, 2007, 2007; White, 2007).

O presente trabalho tem como objetivo compreender como os especialistas, estudiosos da dependência de substâncias psicoativas, consideram o sucesso de um tratamento dessa problemática levando em conta suas vivências, experiências e as suas diferentes abordagens relacionadas ao tema no Brasil.

\section{Metodologia}

Foram utilizados os princípios da metodologia qualitativa, considerando que o objetivo deste trabalho foi investigar a visão dos envolvidos no cuidado e atenção ao usuário de droga, ou seja, obter as suas opiniões, experiências e valores, os quais foram o substrato para esse trabalho (WHO, 1994). A investigação qualitativa proporciona uma visão construtiva-interpretativa do conhecimento científico apresentando a significação da singularidade como nível legitimo de conhecimento (Carrillo, 2004; Minayo \& Sanches, 1993; Gonzalez Rey, 1997; Nogueira-Martins \& Bógus, 2004).

A amostra do estudo foi composta por acadêmicos doutores que desenvolveram trabalhos relacionados ao tema da dependência além da experiência clínica na atenção e cuidado a dependentes.

A construção da amostra foi baseada no princípio da amostra intencional, pois não se busca num estudo qualitativo, a representação estatística e nem a aleatoriedade, ao invés, seleciona-se intencionalmente os participantes que vivenciaram o fenômeno sob estudo, os denominados casos ricos em informação (Victora et al., 2000), procurando dentro da amostra, a maior variedade possível de casos, de forma a contemplar as diferentes perspectivas do fenômeno (Pope et al., 2000).

Utilizou-se como ferramenta para essa pesquisa um questionário eletrônico na plataforma Survey Monkey, através da qual foram envidas perguntas abertas para diversos especialistas. As respostas foram consideradas até que as informações prestadas tornaram-se repetitivas, nesse momento, denominado ponto de saturação teórica, nenhuma nova informação foi identificada (Creswell, 2009). Na prática, o ponto de saturação teórica é definido como a interrupção de inclusão de novos participantes na amostra quando os dados obtidos passam a apresentar, na avaliação do pesquisador, uma certa redundância ou repetição, não sendo considerado relevante continuar com a coleta de dados (Fontanella et al., 2011; Patton, 2002).=

$\mathrm{O}$ recrutamento dos especialistas foi feito utilizando-se a plataforma Lattes do CNPq. Foi utilizada uma amostragem por critérios (de inclusão), ou seja, os especialistas deveriam ter ao menos dois anos de atuação com o tema de dependência, critério que garantiu experiência do entrevistado com o assunto. Além disso, também foi utilizado como critério ser residente no país, apresentar título de doutor com atividades na área de dependência de drogas. Foram então convidados por e-mails e submetidos a um questionário de 13 perguntas abertas criado na plataforma do Survey Monkey como explicado anteriormente. Juntamente com este e-mail foi enviado o TCLE aprovado pelo CEP da UNIFESP. No total, foram enviados 464 e-mails e com um retorno de 13\%. Destes, foram selecionados 55 entrevistados, considerando o ponto de saturação teórica e critérios de inclusão. 
O processo de análise dos resultados seguiu as seguintes etapas:

Análise de Conteúdo: O processo de análise dos resultados foi iniciado identificando-se cada um dos entrevistados com um código alfanumérico, onde a primeira letra do nome foi acoplada ao número de ordem de recebimento do questionário. Os questionários foram lidos e analisados com base nos princípios da análise de conteúdo (Bardin, 2004). Para tanto, após a leitura do material, foram desenvolvidas as etapas de Exploração do Material e Tratamento dos Resultados.

(i) Preparação do material: os questionários foram desmembrados e reagrupados de acordo com o tópico e questão. Esse material deu origem a arquivos independentes para cada item do questionário cada um deles compreendendo respostas que corresponderam a cada integrante da amostra (Bardin, 2004; Minayo \& Sanches, 1993). Para esta preparação do material contou-se com a ajuda dos recursos disponíveis na plataforma do Survey Monkey. A partir desse momento as categorias, em relação às diferentes concepções sobre sucesso do tratamento, foram construídas.

(II) Tratamento dos resultados: Foi utilizada a técnica de triangulação para a análise dos resultados, onde o outro pesquisador (co-autor deste artigo) reconstruiu as categorias identificadas (Patton, 2002). Dessa forma, possibilitou-se a avaliação da convergência das interpretações diminuindo a probabilidade de má interpretação, produzindo resultados mais adequados. Algumas narrativas dos entrevistados são apresentadas em itálico no texto e são identificadas pelo código alfanumérico explicado anteriormente.

Considerações éticas: Este estudo é parte da dissertação de doutorado realizado na Escola Paulista de Medicina da Universidade Federal de São Paulo, através do Programa de Pós Graduação em Saúde Coletiva. Ele foi aprovado pelo Comitê de Ética em Pesquisa da UNIFESP sob número: 0738/2019 e o termo de consentimento livre e esclarecido foi obtido de cada participante da pesquisa e o anonimato preservado.

\section{Resultados}

Entre os componentes da amostra, destacando-se em maior número os profissionais entre 40 e 60 anos de idade.

Em relação à formação profissional houve uma diversidade de oito diferentes categorias profissionais, sendo psicólogos e médicos em maior número, respectivamente $25 \%$ e $20 \%$. Enfermeiros, sociólogos, fonoaudiólogos, terapeutas ocupacionais e educadores/professores também responderam o questionário.

Os profissionais entre 10 a 20 anos de experiência no tema dependência constituíram o grupo de maior representação numérica na amostra (44\%). Os questionários relativos ao intervalo menor que 2 anos não fizeram parte da amostra.

Buscou-se entender como os entrevistados consideravam ter atingido o sucesso em um tratamento de dependência por consumo de substância. Definiram uma série de condições que uma vez alcançadas pelo dependente esses profissionais afirmavam terem obtido sucesso no tratamento.

Essas condições informadas pelos entrevistados dessa amostra foram divididas em cinco categorias; Mudança no padrão de consumo; Qualidade de vida; Reinserção social; Autonomia e Desenvolvimento emocionais.

\section{(i) Mudança no padrão de consumo}

Esta categoria compreende as repostas que apontam como fator determinante para o sucesso do tratamento, um novo padrão de consumo, seja ele a abstinência ou uma nova forma de se relacionar com seu uso. Mais de 39\% dos entrevistados, que responderam essa questão, acreditam que este fator pode apontar o sucesso no tratamento. Dentro dessa categoria foram identificadas duas subcategorias.

Abstinência- Os profissionais nesse caso só admitiam o sucesso com o abandono total da droga.

Manter-se em tratamento com abstinência. Como doença crônica, recaídas são parte da evolução. (I3) 
Não usar mais e se manter abstêmio. (T36)

Diminuição do uso- Uma alteração de consumo em direção à diminuição do consumo, seria um critério de sucesso.

Talvez não somente o não uso mas uma redução do uso já seria um sinal de sucesso. (A41)

Redução da frequência de uso, redução na quantidade de uso, no tempo de intervalo entre o uso..." (D40)

\section{(ii) Qualidade de Vida}

A relevância desta categoria é a importância que os entrevistados dão ao modo como os pacientes passam a lidar com a sua vida, com os cuidados a sua saúde e com as relações interpessoais.

Acredito que o sucesso no tratamento é quando o usuário consegue manter uma qualidade de vida, em que se reduza os riscos à saúde, que ele consiga manter atividades cotidianas. (K28)

Melhora na qualidade de vida pessoal e nas relações com as pessoas e família. Construção de uma vida independente e satisfatória” (A26)

Melhora da qualidade de vida e dos índices de saúde fisica e mental”. (M31)

\section{(iii) Reinserção Social}

A retomada de uma função no trabalho, e das relações com a família e amigos são considerados fatores de extrema importância para o sucesso do tratamento.

Sucesso no tratamento de dependência de substância é o sujeito retomar os compromissos da vida social (relacionamento e trabalho principalmente) que havia deixado em razão da dependência. (R18)

Retomar às atividades sociais, familiares e ocupacionais que foram interrompidas pelo consumo de drogas. Coloca ainda, 1. Renda própria por trabalho 2. Convívio familiar e social. (C27)

\section{(iv) Desenvolvimento Emocional}

Nessa categoria foram reunidas as habilidades que esperam que o dependente adquira e que possam ajudá-lo a uma maturidade emocional. Que possam manejar suas emoções de forma assertiva evitando sabotá-las com o consumo de alguma substância.

Quando a pessoa consegue elaborar projetos/planos e colocar algo em prática. Quando a pessoa consegue tomar algumas decisões pensadas, não impulsivas, pedir ajuda quando precisa, compartilhar seus medos e anseios de modo mais assertivo, acreditar em sua capacidade de enfrentar as adversidades, não se vitimizando e nem minimizando suas fragilidades. (M39)

Observação de comportamentos e atitudes sem drogas que indiquem as capacidades de amar, de enfrentar a realidade e ter um sentido na vida. (W4)

O resgate da autonomia sobre a substância de abuso e a resolução das consequências do transtorno em todas as áreas da vida, o equilíbrio do capital humano. (A11) 


\section{(v) Autonomia}

Esperam que o dependente passe a conduzir a própria vida com autonomia. Segundo esses profissionais, essa conduta seria uma garantia de sucesso do tratamento.

Gerenciar suas escolhas perante família, amigos e outras esferas da vida, e consegue exercer sua autonomia para fazer escolhas com responsabilidade, e assumir consequências, com direito a erros e acertos. (M39)

Que o paciente consiga o máximo de autonomia possível para ela no que se refere ao uso de drogas, relações interpessoais e demais dimensões de sua vida. (M16)

\section{Discussão}

Os profissionais deste estudo esperam, como resultados dos seus esforços, que os dependentes de substâncias psicoativas consigam ficar abstinentes, terem controle sobre o uso dessas substâncias, desenvolverem autonomia, reestruturem suas vidas em diversos aspectos, como relacionamento familiar e trabalho, fazerem escolhas com responsabilidade. É possível notar que não há um padrão entre esses profissionais na definição de sucesso de tratamento, pelo contrário, apontam para uma diversidade de aspectos esperados. E ainda, as condições que os profissionais deste estudo impõem, para serem alcançadas pelos dependentes para obtenção de um resultado de sucesso no tratamento, são audaciosas e de difícil alcance pois são descoladas da realidade do dependente. Nessas expectativas não estão embutidas outras variáveis que interferem neste sucesso como por exemplo, a existência de uma família não amistosa, do preconceito e estigma que recaem sobre os dependentes, a falta de credibilidade que muitos demonstram com dependentes de substâncias ativas etc. Fatores que podem impedir que o indivíduo se sinta um caso de sucesso, apesar dos esforços empregados nesse sentido.

Porém, destaca-se que as condições de sucesso do tratamento encontradas neste estudo estão de acordo com aquelas encontradas pela comunidade científica. Tanto em relação ao conteúdo como em diversidade de aspectos (Inanlou et al., 2020).

A seguir são feitas reflexões sobre algumas das condições comentadas pelos profissionais que são peças na mensuração para o sucesso do tratamento.

Família: Alguns profissionais apontam que a reconexão com a família pode ser um fator preditivo de sucesso. Porém, a família nem sempre é esse fator determinante. Autores afirmam que $75 \%$ de recaídas ocorrem, entre outras causas, devido a falta do apoio social e familiar (Hosseini et al., 2014; Kassani et al., 2015). Esse achado mostra que a família nem sempre tem um papel benéfico ou protetivo. Marchi et al., (2017) concluíram que famílias de usuários de crack, por exemplo, tem vínculos frágeis entre seus membros e laços rompidos de afeto. A existência de desarmonia, discussões e violência podem ser fatores que desencadeiam recaídas.

A abstinência isoladamente, como comentada pelos profissionais, é uma condição complexa de ser mantida considerando que as pistas ambientais desencadeiam fissura que podem levar à recaídas, portanto, dependendo do entorno do local que vive, o estado de abstinência é bastante difícil de ser mantido pelo dependente (Bruehl et al., 2006; Chaves et al., 2011).

Retomar trabalho e conduzir a vida com autonomia são condições que sofrem a interferência negativa da sociedade. A rejeição social ao uso de droga é bastante enraizada na sociedade tendo desdobramentos na percepção sobre dependentes, os quais são vistos como incapazes de auto-controle e responsáveis pelo seu próprio comportamento (Corrigan et al., 2009; Lee e Boeri, 2017). Essa visão pode estar ligada ao discurso do modelo moral o qual rotula qualquer uso de droga como errado e ilegal o que faz dessas pessoas, que utilizam ou utilizaram substâncias, marginais pelo ato de ilegalidade praticado, gerando desconfiança (Hawk et al, 2017). Esse olhar pode afetar o processo de recuperação e reinserção na sociedade. 
A categoria de desenvolvimento emocional é constituída de uma subjetividade difícil de ser alcançada, como por exemplo, ser capaz de amar, tomar decisões não impulsivas, não se vitimizar, resolver todas as áreas de sua vida, ter um sentido na vida. Tais conquistas fazem parte dos anseios da humanidade que as buscam por toda vida (Sommerhalder, 2010).

Por fim, a categoria autonomia aponta para expectativas bastante exigentes, como conduzir a vida com independência. Espera-se desses indivíduos características que a população em geral leva muito tempo para adquirir, sendo que na grande maioria das vezes, só são alcançadas na maturidade, fazendo parte do ciclo vital de todos os indivíduos (Gonçalves, 2016). Dessa forma impor como condição de sucesso de tratamento expectativas de grande exigência é decretar o fracasso do tratamento.

Nesse sentido, diversos autores buscaram compreender a relação entre as expectativas dos profissionais e bons resultados nos tratamentos (Amanzio \& Benedetti, 1999; Colloca \& Finniss, 2012). Spagnolo et al., (2015) colocam que a relação médico-paciente tem impacto na eficácia terapêutica dos tratamentos farmacológicos. Segundo os autores, as expectativas dos profissionais de saúde interferem no resultado do tratamento podendo contribuir para produzir efeitos placebo e nocebo, que por sua vez afetam o curso da doença e da resposta à terapia.

Leamy et al. (2011) analisando a narrativa de pessoas que afirmaram ter tido sucesso no seu tratamento, identificaram as seguintes definições de sucesso: passar a ter esperança e otimismo com o futuro, estabelecer conexão com a vida, descobrir o seu lugar na vida. Em nenhuma dessas definições está incluída a droga, mas somente as transformações que tiveram como pessoas. O que se pode inferir que o sucesso de um tratamento vai além da abstinência ou da redução do consumo da droga. Outros parâmetros estão em jogo e podem estar subestimados ou mesmo, não considerados como fatores de sucesso.

Concordamos com Laudet, (2007) quando diz que a falta de um consenso sobre o que é sucesso num tratamento acaba fortalecendo o estigma e a discriminação do usuário, pois como visto neste estudo, a diversidade de aspectos levantados como condição para a mensuração de sucesso configura-se numa lista de expectativas, com pouca probabilidade de ser alcançada, mas que é necessária, de acordo com os profissionais participantes desta pesquisa, para legitimação da reintegração do dependente na sociedade.

\section{Conclusão}

Os achados deste estudo demonstraram uma grande diversidade de expectativas dos profissionais de saúde em relação ao sucesso do tratamento empregado em dependência. Várias dessas expectativas são de grande exigência constituindo-se em metas difíceis de serem alcançadas o que pode interferir de forma negativa nos resultados do tratamento. A lista de condições que são impostas pelos profissionais aos dependentes, para que o tratamento seja um sucesso, pode desestimulá-lo a permanecer em tratamento, perpetuando um ciclo de fracassos que leva ao estabelecimento de uma dinâmica perigosa na qual esses dependentes passam a não mais procurar ajuda para tratamento de sua dependência. É urgente e necessário que o conceito de sucesso de tratamento ou recovery seja revisitado por associações de profissionais de saúde e que um posicionamento seja elaborado no qual critérios de sucesso sejam definidos, levando-se em consideração o dependente e todas as variáveis que incidem sobre ele. Nesse sentido, trabalhos científicos enfocando esse tema devem ser desenvolvidos de modo a oferecer subsídios que possam ajudar nessa definição.

\section{Bibliografia}

Amanzio, M., \& Benedetti, F. (1999). Neuropharmacological dissection of placebo analgesia: expectation-activated opioid systems versus conditioningactivated specific subsystems. The Journal of neuroscience: the official journal of the Society for Neuroscience,19(1), 484-494. https://doi.org/10.1523/JNEUROSCI.19-01-00484.1999 
Behere, R. V., Muralidharan, K., \& Benegal, V. (2009). Complementary and alternative medicine in the treatment of substance use disorders--a review of the evidence. Drug and alcohol review, 28(3), 292-300. https://doi.org/10.1111/j.1465-3362.2009.00028.x

Betty Ford Institute Consensus Panel (2007). What is recovery? A working definition from the Betty Ford Institute. Journal of substance abuse treatment, 33(3), 221-228. https://doi.org/10.1016/j.jsat.2007.06.001

Bruehl, A. M., Lende, D. H., Schwartz, M., Sterk, C. E., \& Elifson, K. (2006). Craving and control: methamphetamine users' narratives. Journal of psychoactive drugs, Suppl 3, 385-392. https://doi.org/10.1080/02791072.2006.10400602.

Carrillo, E. R. (2004). Psicología para América Latina. In Psicologia para América Latina (Issue 2). Unión Latinoamericana de Entidades de Psicología (ULAPSI). http://pepsic.bvsalud.org/scielo.php?script=sci_arttext\&pid=S1870-350X2004000200003

Center for Substance Abuse Treatment. (2010). National Summit on Recovery From Substance Use Disorders: Bringing Together the Head and the Heart of Recovery. In Mental Health Services.

Chaves, T. V., Sanchez, Z. M., Ribeiro, L. A., \& Nappo, S. A. (2011). Crack cocaine craving: Behaviors and coping strategies among current and former users. Revista de Saude Publica, 45(6), 1168-1175. https://doi.org/10.1590/S0034-89102011005000066

Colloca, L., \& Finniss, D. (2012). Nocebo effects, patient-clinician communication, and therapeutic outcomes. JAMA, 307(6), 567-568. https://doi.org/10.1001/jama.2012.115

Corrigan, P. W., Larson, J. E., Hautamaki, J., Matthews, A., Kuwabara, S., Rafacz, J., Walton, J., Wassel, A., \& O'Shaughnessy, J. (2009). What lessons do coming out as gay men or lesbians have for people stigmatized by mental illness? Community mental health journal, 45(5), 366-374

Creswell, J. W. (2009). Research design: qualitative, quantitative and mixed methods approaches. Thousand Oaks (3rd ed.). Sage Publications

El-Guebaly N. (2012). The meanings of recovery from addiction: evolution and promises. Journal of addiction medicine, 6(1), 1-9. https://doi.org/10.1097/ADM.0b013e31823ae540

Fontanella, B. J. B., Luchesi, B. M., Saidel, M. G. B., Ricas, J., Turato, E. R., \& Melo, D. G. (2011). Amostragem em pesquisas qualitativas: Proposta de procedimentos para constatar saturação teórica. Cadernos de Saude Publica, 27(2), 389-394. https://doi.org/10.1590/s0102-311x2011000200020

Galanter M. (2007). Spirituality and recovery in 12-step programs: an empirical model. Journal of substance abuse treatment, 33(3), 265-272. https://doi.org/10.1016/j.jsat.2007.04.016

Gonçalves, J. P. (2016). Ciclo vital: início, desenvolvimento e fim da vida humana possíveis contribuições para educadores. Revista Contexto \& Educação, 31(98), 79-110. https://doi.org/10.21527/2179-1309.2016.98.79-110

Gonzalez Rey, F. (1997). Epistemologia cualitativa y subjetividad. EDUC. https://www.estantevirtual.com.br/livros/fernando-gonzalez-rey/epistemologiacualitativa-y-subjetividad/1643878634

Hawk, M., Coulter, R., Egan, J. E., Fisk, S., Reuel Friedman, M., Tula, M., \& Kinsky, S. (2017). Harm reduction principles for healthcare settings. Harm reduction journal, 14(1), 70. https://doi.org/10.1186/s12954-017-0196-4

Hosseini, S., Moghimbeigi, A., Roshanaei, G., \& Momeniarbat, F. (2014). Evaluation of drug abuse relapse event rate over time in frailty model. Osong public health and research perspectives, 5(2), 92-95. https://doi.org/10.1016/j.phrp.2014.02.003

Inanlou, M., Bahmani, B., Farhoudian, A., \& Rafiee, F. (2020). Addiction Recovery: A Systematized Review. Iranian journal of psychiatry, 15(2), $172-181$.

Kassani, A., Niazi, M., Hassanzadeh, J., \& Menati, R. (2015). Survival Analysis of Drug Abuse Relapse in Addiction Treatment Centers. International journal of high risk behaviors \& addiction, 4(3), e23402. https://doi.org/10.5812/ijhrba.23402

Laudet A. B. (2007). What does recovery mean to you? Lessons from the recovery experience for research and practice. Journal of substance abuse treatment, 33(3), 243-256. https://doi.org/10.1016/j.jsat.2007.04.014

Laudet, A. B., \& White, W. (2010). What are your priorities right now? Identifying service needs across recovery stages to inform service development. Journal of substance abuse treatment, 38(1), 51-59. https://doi.org/10.1016/j.jsat.2009.06.00

Leamy, M., Bird, V., Le Boutillier, C., Williams, J., \& Slade, M. (2011). Conceptual framework for personal recovery in mental health: systematic review and narrative synthesis. The British journal of psychiatry: the journal of mental science, 199(6), 445-452. https://doi.org/10.1192/bjp.bp.110.083733

Lee, N., \& Boeri, M. (2017). Managing Stigma: Women Drug Users and Recovery Services. Fusio: the Bentley undergraduate research journal, 1(2), 65-94.

Lopes-Rosa, R., Kessler, F. P., Pianca, T. G., Guimarães, L., Ferronato, P., Pagnussat, E., Moura, H., Pechansky, F., \& von Diemen, L. (2017). Predictors of early relapse among adolescent crack users. Journal of addictive diseases, 36(2), 136-143. https://doi.org/10.1080/10550887.2017.1295670

Marchi, N. C., Scherer, J. N., Pachado, M. P., Guimarães, L. S., Siegmund, G., de Castro, M. N., Halpern, S., Benzano, D., Formigoni, M. L., Cruz, M., Pechansky, F., \& Kessler, F. H. (2017). Crack-cocaine users have less family cohesion than alcohol users. Revista brasileira de psiquiatria (Sao Paulo, Brazil : 1999), 39(4), 346-351. https://doi.org/10.1590/1516-4446-2016-2091

Marlatt, G. (1999). Redução de danos: estratégias práticas para lidar com comportamentos de alto risco. Artmed.

Minayo, C. S. \& Sanches, O. (1993). Quantitativo-Qualitativo: Oposição ou Complementaridade? Quantitative and Qualitative Methods: Opposition or Complementarity? 9(3). http://www.scielo.br/pdf/csp/v9n3/02.pdf

Nogueira-Martins, M. C. F., \& Bógus, C. M. (2004). Considerações sobre a metodologia qualitativa como recurso para o estudo das ações de humanização em saúde. Saúde e Sociedade, 13(3), 44-57. https://doi.org/10.1590/s0104-12902004000300006 
Research, Society and Development, v. 10, n. 14, e187101419999, 2021

(CC BY 4.0) | ISSN 2525-3409 | DOI: http://dx.doi.org/10.33448/rsd-v10i14.19999

Oliveira, L. G., \& Nappo, S. A. (2008). Caracterização da cultura de crack na cidade de São Paulo: padrão de uso controlado [Characterization of the crack cocaine culture in the city of São Paulo: a controlled pattern of use]. Revista de saude publica,42(4), 664-671. https://doi.org/10.1590/s003489102008005000039

Patton, M. Q. (2002). Qualitative research and evaluation methods. Sage Publications.

Pope, C., Ziebland, S., \& Mays, N. (2000). Qualitative research in health care. Analysing qualitative data. BMJ (Clinical research ed.), 320(7227), 114-116. https://doi.org/10.1136/bmj.320.7227.114

Ribeiro, C. T., \& Fernandes, A. H. (2013). Tratamentos para usuários de drogas: possibilidades, desafios e limites da articulação entre as propostas da redução de danos e da psicanálise. Analytica: Revista de Psicanálise, 2(2). http://pepsic.bvsalud.org/scielo.php?script=sci_arttext\&pid=S2316-51972013000100003

Rigotto, S. D., \& Gomes, W. B. (2002). Contextos de abstinência e de recaída na recuperação da dependência química. Psicologia: Teoria e Pesquisa, 18(1), 95-106. https://doi.org/10.1590/S0102-37722002000100011

Sadeghieh, S., Azami, A., Amani, F., \& Barak, M. (2004). Reviewing the causes of recurred addiction in patients who referred to centers introduced of Tehran welfare. Ardabil Med Univ J, 3(4), 36-40. https://scholar.google.com/citations?view_op=view_citation\&hl=en\&user=UvNAXRYAAAAJ\&cstart=20\&pag esize $=80 \&$ sortby=pubdate\&citation_for_view=UvNAXRYAAAAJ:3fE2CSJIrl8C

Sommerhalder, C. (2010). Sentido de vida na fase adulta e velhice. Psicologia: Reflexão e Crítica, 23(2), 270-277. https://doi.org/10.1590/S010279722010000200009

Spagnolo, P. A., Colloca, L., \& Heilig, M. (2015). The role of expectation in the therapeutic outcomes of alcohol and drug addiction treatments. Alcohol and alcoholism (Oxford, Oxfordshire), 50(3), 282-285. https://doi.org/10.1093/alcalc/agv015

Tomko, R. L., Bountress, K. E., \& Gray, K. M. (2016). Personalizing substance use treatment based on pre-treatment impulsivity and sensation seeking: A review. Drug and alcohol dependence, 167, 1-7. https://doi.org/10.1016/j.drugalcdep.2016.07.022

White W. L. (2007). Addiction recovery: its definition and conceptual boundaries. Journal of substance abuse treatment, 33(3), 229-241. https://doi.org/10.1016/j.jsat.2007.04.015

WHO - World Health Organization. (1994). Qualitative research for health programmes. World Health Organization. http://apps.who.int/iris/bitstream/10665/62315/1/WHO_MNH_PSF_94.3.pdf

World Drug Report. (2021). UNDOC - United Nations Office on Drugs and Crime. United Nations publication. https://www.unodc.org/res/wdr2021/field/WDR21_Booklet_1.pdf.

Yang, M., Mamy, J., Gao, P., \& Xiao, S. (2015). From Abstinence to Relapse: A Preliminary Qualitative Study of Drug Users in a Compulsory Drug Rehabilitation Center in Changsha, China. PloS one, 10(6), e0130711. https://doi.org/10.1371/journal.pone.0130711 